\title{
Frame-based Average Sampling in Multiply Generated Shift-invariant Subspaces of Mixed Lebesgue Spaces
}

\author{
Yingchun Jiang and Jiao Li*
}

Abstract. In this paper, we mainly discuss the nonuniform average sampling and reconstruction in multiply generated shift-invariant subspaces

$$
\begin{aligned}
& V_{p, q}\left(\Phi_{r}\right) \\
= & \left\{\sum_{k_{1} \in \mathbf{Z}} \sum_{k_{2} \in \mathbf{Z}^{d}} c^{T}\left(k_{1}, k_{2}\right) \Phi_{r}\left(\cdot-k_{1}, \cdot-k_{2}\right):\left(c\left(k_{1}, k_{2}\right)\right)_{\left(k_{1}, k_{2}\right) \in \mathbf{Z} \times \mathbf{Z}^{d}} \in\left(\ell^{p, q}\left(\mathbf{Z} \times \mathbf{Z}^{d}\right)\right)^{r}\right\}
\end{aligned}
$$

of mixed Lebesgue spaces $L^{p, q}\left(\mathbf{R} \times \mathbf{R}^{d}\right), 1 \leq p, q \leq \infty$, where $\Phi_{r}=\left(\varphi_{1}, \varphi_{2}, \ldots, \varphi_{r}\right)^{T}$ with $\varphi_{i} \in L^{p, q}\left(\mathbf{R} \times \mathbf{R}^{d}\right)$ and $c=\left(c_{1}, c_{2}, \ldots, c_{r}\right)^{T}$ with $c_{i} \in \ell^{p, q}\left(\mathbf{Z} \times \mathbf{Z}^{d}\right), i=1,2, \ldots, r$, under the assumption that the family $\left\{\varphi_{i}\left(x-k_{1}, y-k_{2}\right):\left(k_{1}, k_{2}\right) \in \mathbf{Z} \times \mathbf{Z}^{d}, 1 \leq\right.$ $i \leq r\}$ constitutes a $(p, q)$-frame of $V_{p, q}\left(\Phi_{r}\right)$. First, iterative approximation projection algorithms for two kinds of average sampling functionals are established. Then, we estimate the convergence rates of the corresponding algorithms.

\section{Introduction}

Mixed Lebesgue spaces were firstly introduced in [4], although the initial realization of their necessity goes back to [12]. In the last years, there are many groups of people working on mixed Lebesgue spaces from the viewpoint of harmonic analysis and operator theory, refer to $5-8,10,11,13,16,21,22,26]$ and references therein.

For $1 \leq p, q \leq \infty$, the mixed Lebesgue space $L^{p, q}\left(\mathbf{R} \times \mathbf{R}^{d}\right)$ denotes the Banach space of all functions $f$ such that

$$
\|f\|_{L^{p, q}\left(\mathbf{R} \times \mathbf{R}^{d}\right)}=\|\| f\left(x_{1}, x_{2}\right)\left\|_{L_{x_{2}}^{q}\left(\mathbf{R}^{d}\right)}\right\|_{L_{x_{1}}^{p}(\mathbf{R})}<\infty .
$$

Similarly, $\ell^{p, q}=\ell^{p, q}\left(\mathbf{Z} \times \mathbf{Z}^{d}\right)$ is the Banach space of all complex valued sequences $c=$ $\left(c\left(k_{1}, k_{2}\right)\right)_{\left(k_{1}, k_{2}\right) \in \mathbf{Z} \times \mathbf{Z}^{d}}$ such that

$$
\|c\|_{\ell^{p, q}}=\|\| c\left(k_{1}, k_{2}\right)\left\|_{\ell_{k_{2}}^{q}\left(\mathbf{Z}^{d}\right)}\right\|_{\ell_{k_{1}}^{p}(\mathbf{Z})}<\infty
$$

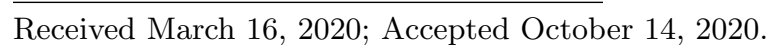

Communicated by Xiang Fang.

2020 Mathematics Subject Classification. 94A20, 42C40.

Key words and phrases. average sampling, $(p, q)$-frame, shift-invariant subspace, mixed Lebesgue space, approximation projection algorithm.

*Corresponding author. 
Sampling plays an important role in signal and information processing, and sampling theory in shift-invariant subspaces of classical Lebesgue spaces $L^{p}\left(\mathbf{R}^{d}\right)$ had been generally studied in the past years, such as $[1,3,9,28,29$. Since mixed Lebesgue space is a suitable tool for modeling and measuring time variant signals due to its separate integrability for different variables, some sampling results in bandlimited subspaces, shift-invariant subspaces and reproducing kernel subspaces of mixed Lebesgue spaces $L^{p, q}\left(\mathbf{R} \times \mathbf{R}^{d}\right)$ are given recently $17,18,20,23,24,27,30,31$. However, the existed works in shift-invariant spaces always assume that the family $\left\{\varphi_{i}\left(x-k_{1}, y-k_{2}\right):\left(k_{1}, k_{2}\right) \in \mathbf{Z} \times \mathbf{Z}^{d}, 1 \leq i \leq r\right\}$ has $(p, q)$-stability [25], instead of a possibly redundant frame.

In this paper, we will study the average sampling and reconstruction problem in the multiply generated shift-invariant subspaces

$$
\begin{aligned}
& V_{p, q}\left(\Phi_{r}\right) \\
= & \left\{\sum_{k_{1} \in \mathbf{Z}} \sum_{k_{2} \in \mathbf{Z}^{d}} c^{T}\left(k_{1}, k_{2}\right) \Phi_{r}\left(\cdot-k_{1}, \cdot-k_{2}\right):\left(c\left(k_{1}, k_{2}\right)\right)_{\left(k_{1}, k_{2}\right) \in \mathbf{Z} \times \mathbf{Z}^{d}} \in\left(\ell^{p, q}\left(\mathbf{Z} \times \mathbf{Z}^{d}\right)\right)^{r}\right\}
\end{aligned}
$$

of mixed Lebesgue spaces $L^{p, q}\left(\mathbf{R} \times \mathbf{R}^{d}\right), 1 \leq p, q \leq \infty$, where $T$ denotes the transpose, $\Phi_{r}=$ $\left(\varphi_{1}, \varphi_{2}, \ldots, \varphi_{r}\right)^{T}$ with $\varphi_{i} \in L^{p, q}\left(\mathbf{R} \times \mathbf{R}^{d}\right)$ and $c=\left(c_{1}, c_{2}, \ldots, c_{r}\right)^{T}$ with $c_{i} \in \ell^{p, q}\left(\mathbf{Z} \times \mathbf{Z}^{d}\right)$, $i=1,2, \ldots, r$, based on the following assumptions:

(A1) The family $\left\{\varphi_{i}\left(\cdot-k_{1}, \cdot-k_{2}\right):\left(k_{1}, k_{2}\right) \in \mathbf{Z} \times \mathbf{Z}^{d}, 1 \leq i \leq r\right\}$ is a $(p, q)$-frame for $V_{p, q}\left(\Phi_{r}\right)$, which is defined and characterized in 19 .

Definition 1.1. The family $\left\{\varphi_{i}\left(x_{1}-k_{1}, x_{2}-k_{2}\right): i=1, \ldots, r,\left(k_{1}, k_{2}\right) \in \mathbf{Z} \times \mathbf{Z}^{d}\right\}$ is called a $(p, q)$-frame of $V_{p, q}\left(\Phi_{r}\right)$ if $\varphi_{i} \in L^{p^{\prime}, q^{\prime}}\left(\mathbf{R} \times \mathbf{R}^{d}\right)$ and there exists a positive constant $A \geq 1$ (depending on $p, q$ and $\Phi_{r}$ ) such that for any $f \in V_{p, q}\left(\Phi_{r}\right)$,

$$
A^{-1}\|f\|_{L^{p, q}} \leq \sum_{i=1}^{r}\left\|\left\{\left\langle f, \varphi_{i}\left(\cdot-k_{1}, \cdot-k_{2}\right)\right\rangle\right\}_{\left(k_{1}, k_{2}\right) \in \mathbf{Z} \times \mathbf{Z}^{d}}\right\|_{\ell^{p, q}} \leq A\|f\|_{L^{p, q}},
$$

where $\langle\cdot\rangle$ denotes the dual product between $L^{p, q}\left(\mathbf{R} \times \mathbf{R}^{d}\right)$ and $L^{p^{\prime}, q^{\prime}}\left(\mathbf{R} \times \mathbf{R}^{d}\right), p^{\prime}$ and $q^{\prime}$ are the conjugate numbers of $p$ and $q$, respectively.

(A2) The generators $\varphi_{i}, 1 \leq i \leq r$ are continuous and belong to the mixed Wiener amalgam space $W\left(L^{1,1}\right)=W\left(L^{1,1}\right)\left(\mathbf{R} \times \mathbf{R}^{d}\right)$ whose general forms are defined as all measurable functions satisfying

$$
\|f\|_{W\left(L^{p, q}\right)}=\left(\sum_{k_{1} \in \mathbf{Z}^{x_{1}} \in[0.1]} \sup _{k_{2} \in \mathbf{Z}^{d}}\left(\sum_{x_{2} \in[0.1]^{d}}\left|f\left(x_{1}+k_{1}, x_{2}+k_{2}\right)\right|^{q}\right)^{p / q}\right)^{1 / p}<\infty
$$

for $1 \leq p, q<\infty$. The cases $p=\infty$ or $q=\infty$ obey the usual adjustment. 
(A3) We assume that there exists some $\delta_{0}>0$ such that $\operatorname{osc}_{\left(\delta_{0}, x\right)}\left(\varphi_{i}\right) \in W\left(L^{1,1}\right)$ for all $1 \leq i \leq r$. Here, $\operatorname{osc}_{\left(\delta_{0}, x\right)}(\varphi)$ is the partial oscillation (or modulus of continuity) about variable $x$ defined by

$$
\operatorname{osc}_{(\delta, x)}(\varphi)(x, y)=\sup _{\left|x^{\prime}\right| \leq \delta}\left|\varphi\left(x+x^{\prime}, y\right)-\varphi(x, y)\right|
$$

Moreover, we define the partial oscillation about the variable $y$ as

$$
\operatorname{osc}_{(\delta, y)}(f)(x, y)=\sup _{\left|y^{\prime}\right| \leq \delta}\left|f\left(x, y+y^{\prime}\right)-f(x, y)\right|
$$

and the oscillation

$$
\operatorname{osc}_{\delta}(f)(x, y)=\sup _{\left|x^{\prime}\right| \leq \delta,\left|y^{\prime}\right| \leq \delta}\left|f\left(x+x^{\prime}, y+y^{\prime}\right)-f(x, y)\right|
$$

In fact, if $\varphi$ belongs to the classical Wiener amalgam space $W\left(L^{1}\right)\left(\mathbf{R}^{d+1}\right)$ defined by

$$
\|\varphi\|_{W\left(L^{1}\right)}=\sum_{k_{1} \in \mathbf{Z}} \sum_{k_{2} \in \mathbf{Z}^{d}} \sup _{(x, y) \in[0,1]^{d+1}}\left|\varphi\left(x+k_{1}, y+k_{2}\right)\right|<\infty,
$$

then we can obtain

$$
\begin{aligned}
& \left\|\operatorname{osc}_{\left(\delta_{0}, x\right)}(\varphi)\right\|_{W\left(L^{1,1}\right)} \\
= & \sum_{k_{1} \in \mathbf{Z}} \sup _{x \in[0,1]} \sum_{k_{2} \in \mathbf{Z}^{d}} \sup _{y \in[0,1]^{d}} \sup _{\left|x^{\prime}\right| \leq \delta_{0}}\left|\varphi\left(x+x^{\prime}+k_{1}, y+k_{2}\right)-\varphi\left(x+k_{1}, y+k_{2}\right)\right| \\
\leq & \|\varphi\|_{W\left(L^{1,1}\right)}+\sum_{k_{1} \in \mathbf{Z}} \sum_{k_{2} \in \mathbf{Z}^{d}} \sup _{(x, y) \in[0,1]^{d+1}} \sup _{\left|x^{\prime}\right| \leq \delta_{0}}\left|\varphi\left(x+x^{\prime}+k_{1}, y+k_{2}\right)\right| \\
\leq & \left(4+2\left[\delta_{0}\right]\right)\|\varphi\|_{W\left(L^{1}\right)}<\infty .
\end{aligned}
$$

With the requirements (A1) and (A2), we know from [19] that

(i) The space $V_{p, q}\left(\Phi_{r}\right)$ is a closed linear subspace of $L^{p, q}\left(\mathbf{R} \times \mathbf{R}^{d}\right)$ and there exists a positive constant $B \geq 1$ (depending on $p, q$ and $\Phi_{r}$ ) such that

$$
B^{-1}\|f\|_{L^{p, q}} \leq \inf _{f=\sum_{i=1}^{r} \varphi_{i} *^{\prime} c_{i}} \sum_{i=1}^{r}\left\|c_{i}\right\|_{\ell^{p, q}} \leq B\|f\|_{L^{p, q}}, \quad \forall f \in V_{p, q}\left(\Phi_{r}\right),
$$

where $\varphi_{i} *^{\prime} c_{i}=\sum_{k_{1} \in \mathbf{Z}} \sum_{k_{2} \in \mathbf{Z}^{d}} c_{i}\left(k_{1}, k_{2}\right) \varphi_{i}\left(\cdot-k_{1}, \cdot-k_{2}\right)$.

(ii) There exists $\widetilde{\varphi}_{1}, \ldots, \widetilde{\varphi}_{r} \in W\left(L^{1,1}\right) \cap V_{p, q}\left(\Phi_{r}\right)$ such that for every $f \in V_{p, q}\left(\Phi_{r}\right)$,

$$
\begin{aligned}
f & =\sum_{i=1}^{r} \sum_{k_{1} \in \mathbf{Z}} \sum_{k_{2} \in \mathbf{Z}^{d}}\left\langle f, \widetilde{\varphi}_{i}\left(\cdot-k_{1}, \cdot-k_{2}\right)\right\rangle \varphi_{i}\left(\cdot-k_{1}, \cdot-k_{2}\right) \\
& =\sum_{i=1}^{r} \sum_{k_{1} \in \mathbf{Z}} \sum_{k_{2} \in \mathbf{Z}^{d}}\left\langle f, \varphi_{i}\left(\cdot-k_{1}, \cdot-k_{2}\right)\right\rangle \widetilde{\varphi}_{i}\left(\cdot-k_{1}, \cdot-k_{2}\right) .
\end{aligned}
$$


The paper is organized as follows. In Section 2, we give some lemmata which will provide the theoretical basis for the subsequent sections. In Section 3, iterative approximation projection algorithms for two kinds of average sampling schemes are given. In Section 4, we estimate the corresponding convergence rates.

\section{Some lemmata}

In this section, we will give some lemmata which will be used in the subsequent sections.

Lemma 2.1. If $f \in W\left(L^{1,1}\right)$ and $g \in L^{1}\left(\mathbf{R}^{d+1}\right)$ then $f * g \in W\left(L^{1,1}\right)$ and

$$
\|f * g\|_{W\left(L^{1,1}\right)} \leq 2^{d+1}\|f\|_{W\left(L^{1,1}\right)}\|g\|_{L^{1}} .
$$

Proof. By the definition of convolution, we can obtain

$$
\begin{aligned}
& \|f * g\|_{W\left(L^{1,1}\right)} \\
\leq & \sum_{k_{1} \in \mathbf{Z}} \sup _{x \in[0,1]} \sum_{k_{2} \in \mathbf{Z}^{d}} \sup _{y \in[0,1]^{d}} \int_{\mathbf{R}} \int_{\mathbf{R}^{d}}\left|f\left(x+k_{1}-s, y+k_{2}-t\right)\right||g(s, t)| d t d s \\
\leq & \sum_{\ell_{1} \in \mathbf{Z}} \sum_{\ell_{2} \in \mathbf{Z}^{d}} \int_{0}^{1} \int_{{ }_{[0,1]^{d}}} \sum_{k_{1} \in \mathbf{Z}^{\prime}} \sup _{x \in[0,1]} \sum_{k_{2} \in \mathbf{Z}^{d}} \sup _{y \in[0,1]^{d}}\left|f\left(x+k_{1}-s-\ell_{1}, y+k_{2}-t-\ell_{2}\right)\right| \\
& \times\left|g\left(s+\ell_{1}, t+\ell_{2}\right)\right| d t d s \\
\leq & \sum_{\ell_{1} \in \mathbf{Z}} \sum_{\ell_{2} \in \mathbf{Z}^{d}} \sup _{s \in[0,1]} \sup _{t \in[0,1]^{d}}\left(\sum_{k_{1} \in \mathbf{Z}^{x \in[0,1]}} \sum_{k_{2} \in \mathbf{Z}^{d}} \sup _{y \in[0,1]^{d}}\left|f\left(x+k_{1}-s-\ell_{1}, y+k_{2}-t-\ell_{2}\right)\right|\right) \\
& \times \int_{0}^{1} \int_{[0,1]^{d}}\left|g\left(s+\ell_{1}, t+\ell_{2}\right)\right| d t d s \\
\leq & \sum_{\ell_{1} \in \mathbf{Z}} \sum_{\ell_{2} \in \mathbf{Z}^{d}}\left(\sum_{k_{1} \in \mathbf{Z}^{x \in[0,1]}} \sup _{s \in[0,1]} \sum_{k_{2} \in \mathbf{Z}^{d}} \sup _{y \in[0,1]^{d}} \sup _{t \in[0,1]^{d}}\left|f\left(x+k_{1}-s, y+k_{2}-t\right)\right|\right) \\
& \times \int_{0}^{1} \int_{[0,1]^{d}}\left|g\left(s+\ell_{1}, t+\ell_{2}\right)\right| d t d s \\
\leq & 2^{d+1}\|f\|_{W\left(L^{1,1}\right)}\|g\|_{L^{1}} .
\end{aligned}
$$

This completes the proof.

Lemma 2.2. 24, Theorem 3.4] Suppose that $1 \leq p, q \leq \infty$ and $g \in W\left(L^{1,1}\right)\left(\mathbf{R}^{d+1}\right)$. Then for any $f \in L^{p, q}\left(\mathbf{R}^{d+1}\right)$, the sequence

$$
d\left(k_{1}, k_{2}\right)=\int_{\mathbf{R}} \int_{\mathbf{R}^{d}} f\left(x_{1}, x_{2}\right) \overline{g\left(x_{1}-k_{1}, x_{2}-k_{2}\right)} d x_{1} d x_{2}
$$

belongs to $\ell^{p, q}\left(\mathbf{Z} \times \mathbf{Z}^{d}\right)$ and $\|d\|_{\ell^{p, q}} \leq\|f\|_{L^{p, q}}\|g\|_{W\left(L^{1,1}\right)}$. 
Hence, the operator $P$ defined by

$$
P f=\sum_{i=1}^{r} \sum_{k_{1} \in \mathbf{Z}} \sum_{k_{2} \in \mathbf{Z}^{d}}\left\langle f, \widetilde{\varphi}_{i}\left(\cdot-k_{1}, \cdot-k_{2}\right)\right\rangle \varphi_{i}\left(\cdot-k_{1}, \cdot-k_{2}\right)
$$

is a bounded projection from $L^{p, q}\left(\mathbf{R} \times \mathbf{R}^{d}\right)$ onto $V_{p, q}\left(\Phi_{r}\right)$.

Lemma 2.3. 24, Theorem 3.1] Assume that $1 \leq p, q \leq \infty$ and $\varphi \in W\left(L^{1,1}\right)$. Then for any $c \in \ell^{p, q}\left(\mathbf{Z} \times \mathbf{Z}^{d}\right)$, the function $f=\sum_{k_{1} \in \mathbf{Z}} \sum_{k_{2} \in \mathbf{Z}^{d}} c\left(k_{1}, k_{2}\right) \varphi\left(\cdot-k_{1}, \cdot-k_{2}\right)$ belongs to $L^{p, q}\left(\mathbf{R} \times \mathbf{R}^{d}\right)$ and

$$
\|f\|_{L^{p, q}} \leq\|c\|_{\ell^{p, q}}\|\varphi\|_{W\left(L^{1,1}\right)}
$$

Lemma 2.4. Suppose that $\varphi_{i} \in W\left(L^{1,1}\right)$ for all $1 \leq i \leq r$ and that $\left\{\varphi_{i}\left(\cdot-k_{1}, \cdot-k_{2}\right)\right.$ : $\left.\left(k_{1}, k_{2}\right) \in \mathbf{Z} \times \mathbf{Z}^{d}, 1 \leq i \leq r\right\}$ is a $(p, q)$-frame for $V_{p, q}\left(\Phi_{r}\right)$, then for any $f \in V_{p, q}\left(\Phi_{r}\right)$, one has

$$
\|f\|_{L^{p, q}} \leq\|f\|_{W\left(L^{p, q}\right)} \leq B\left(\max _{1 \leq i \leq r}\left\|\varphi_{i}\right\|_{W\left(L^{1,1}\right)}\right)\|f\|_{L^{p, q}}
$$

Proof. Suppose that $f\left(x_{1}, x_{2}\right)=\sum_{i=1}^{r} f_{i}\left(x_{1}, x_{2}\right)$ with

$$
f_{i}\left(x_{1}, x_{2}\right)=\sum_{j_{1} \in \mathbf{Z}} \sum_{j_{2} \in \mathbf{Z}^{d}} c_{i}\left(j_{1}, j_{2}\right) \varphi_{i}\left(x_{1}-j_{1}, x_{2}-j_{2}\right) .
$$

Write $b_{x_{1}+k_{1}-j_{1}}(\cdot)=\sup _{x_{2} \in[0,1]^{d}}\left|\varphi_{i}\left(x_{1}+k_{1}-j_{1}, x_{2}+\cdot\right)\right|$ and $c_{j_{1}}(\cdot)=c_{i}\left(j_{1}, \cdot\right)$. Then

$$
\begin{aligned}
& \left(\sum_{k_{2} \in \mathbf{Z}^{d}} \sup _{x_{2} \in[0,1]^{d}}\left|f_{i}\left(x_{1}+k_{1}, x_{2}+k_{2}\right)\right|^{q}\right)^{1 / q} \\
\leq & \left(\sum_{k_{2} \in \mathbf{Z}^{d}}\left(\sum_{j_{1} \in \mathbf{Z}} c_{j_{1}} * b_{x_{1}+k_{1}-j_{1}}\left(k_{2}\right)\right)^{q}\right)^{1 / q} \\
\leq & \sum_{j_{1} \in \mathbf{Z}}\left\|c_{j_{1}} * b_{x_{1}+k_{1}-j_{1}}\right\|_{\ell^{q}} \leq \sum_{j_{1} \in \mathbf{Z}}\left\|c_{j_{1}}\right\|_{\ell_{j_{2}}^{q}}\left\|b_{x_{1}+k_{1}-j_{1}}\right\|_{\ell_{j_{2}}^{1}} .
\end{aligned}
$$

Let $a_{j_{1}}=\left\|c_{j_{1}}\right\|_{\ell_{j_{2}}^{q}}$ and $d_{k_{1}-j_{1}}=\sup _{x_{1} \in[0,1]}\left\|b_{x_{1}+k_{1}-j_{1}}\right\|_{\ell_{j_{2}}^{1}}$. Then

$$
\begin{aligned}
& \left(\sum_{k_{1} \in \mathbf{Z}} \sup _{x_{1} \in[0,1]}\left(\sum_{j_{1} \in \mathbf{Z}}\left\|c_{j_{1}}\right\|_{\ell_{j_{2}}^{q}}\left\|b_{x_{1}+k_{1}-j_{1}}\right\|_{\ell_{j_{2}}^{1}}\right)^{p}\right)^{1 / p} \\
\leq & \|a * d\|_{\ell^{p}} \leq\|a\|_{\ell_{j_{1}}^{p}}\|d\|_{\ell_{j_{1}}^{1}}=\left\|c_{i}\right\|_{\ell^{p}, q}\left\|\varphi_{i}\right\|_{W\left(L^{1,1}\right)} .
\end{aligned}
$$


This together with 2.2 obtains $\left\|f_{i}\right\|_{W\left(L^{p, q}\right)} \leq\left\|c_{i}\right\|_{\ell^{p, q}}\left\|\varphi_{i}\right\|_{W\left(L^{1,1}\right)}$. Then it follows from (1.1) that

$$
\begin{aligned}
\|f\|_{W\left(L^{p, q}\right)} \leq \sum_{i=1}^{r}\left\|f_{i}\right\|_{W\left(L^{p, q}\right)} & \leq\left(\sum_{i=1}^{r}\left\|c_{i}\right\|_{\ell^{p, q}}\right)\left(\max _{1 \leq i \leq r}\left\|\varphi_{i}\right\|_{W\left(L^{1,1}\right)}\right) \\
& \leq B\left(\max _{1 \leq i \leq r}\left\|\varphi_{i}\right\|_{W\left(L^{1,1}\right)}\right)\|f\|_{L^{p, q}},
\end{aligned}
$$

which is the right-hand side of (2.1). The left side can be easily verified.

Note that $W\left(L^{p, q}\right) \subset L^{\infty, \infty}\left(\mathbf{R} \times \mathbf{R}^{d}\right)$. Then Lemma 2.4 shows that the convergence in

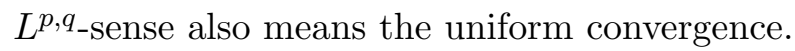

Lemma 2.5. Suppose that $\varphi \in W\left(L^{1,1}\right)$, then $\operatorname{osc}_{(\delta, y)}(\varphi) \in W\left(L^{1,1}\right)$ for any $\delta>0$, and

$$
\left\|\operatorname{osc}_{(\delta, y)}(\varphi)\right\|_{W\left(L^{1,1}\right)} \leq\left(1+(2[\delta]+3)^{d}\right)\|\varphi\|_{W\left(L^{1,1}\right)} .
$$

Proof. For any $\delta>0$, it follows from the definition of $W\left(L^{1,1}\right)$-norm that

$$
\begin{aligned}
& \left\|\operatorname{osc}_{(\delta, y)}(\varphi)\right\|_{W\left(L^{1,1}\right)} \\
\leq & \|\varphi\|_{W\left(L^{1,1}\right)}+\sum_{k_{1} \in \mathbf{Z}} \sup _{x_{1} \in[0,1]} \sum_{k_{2} \in \mathbf{Z}^{d}} \sup _{x_{2} \in[0,1]^{d}} \sup _{\left|y_{2}\right| \leq \delta}\left|\varphi\left(x_{1}+k_{1}, x_{2}+k_{2}+y_{2}\right)\right| \\
\leq & \|\varphi\|_{W\left(L^{1,1}\right)}+\sum_{k_{1} \in \mathbf{Z}} \sup _{x_{1} \in[0,1]} \sum_{k_{2} \in \mathbf{Z}^{d} x_{2} \in[-[\delta]-1,[\delta]+2]^{d}}\left|\varphi\left(x_{1}+k_{1}, x_{2}+k_{2}\right)\right| \\
\leq & \left(1+(2[\delta]+3)^{d}\right)\|\varphi\|_{W\left(L^{1,1}\right)} .
\end{aligned}
$$

Finally, the desired result follows from $\varphi \in W\left(L^{1,1}\right)$.

Lemma 2.6. If $\varphi \in W\left(L^{1,1}\right)$ is continuous and $\operatorname{osc}_{\left(\delta_{0}, x\right)}(\varphi) \in W\left(L^{1,1}\right)$ for some $\delta_{0}>0$, then

$(2.3)\left\|\operatorname{osc}_{\delta_{0}}(\varphi)\right\|_{W\left(L^{1,1}\right)} \leq\left(2+\left(2\left[\delta_{0}\right]+3\right)^{d}\right)\|\varphi\|_{W\left(L^{1,1}\right)}+\left(2\left[\delta_{0}\right]+3\right)^{d}\left\|\operatorname{osc}_{\left(\delta_{0}, x\right)}(\varphi)\right\|_{W\left(L^{1,1}\right)}$ and $\lim _{\delta \rightarrow 0}\left\|\operatorname{osc}_{\delta}(\varphi)\right\|_{W\left(L^{1,1}\right)}=0$.

Proof. Since $\varphi \in W\left(L^{1,1}\right)$ and $\operatorname{osc}_{\left(\delta_{0}, x\right)}(\varphi) \in W\left(L^{1,1}\right)$, this together with Lemma 2.5 obtains

$$
\begin{aligned}
& \left\|\operatorname{osc}_{\delta_{0}}(\varphi)\right\|_{W\left(L^{1,1}\right)} \\
= & \sum_{k_{1} \in \mathbf{Z}} \sup _{x \in[0,1]} \sum_{k_{2} \in \mathbf{Z}^{d}} \sup _{y \in[0,1]^{d}} \sup _{\left|x^{\prime}\right| \leq \delta_{0},\left|y^{\prime}\right| \leq \delta_{0}}\left|\varphi\left(x+x^{\prime}+k_{1}, y+y^{\prime}+k_{2}\right)-\varphi\left(x+k_{1}, y+k_{2}\right)\right| \\
\leq & \|\varphi\|_{W\left(L^{1,1}\right)}+\left\|\operatorname{osc}_{\left(\delta_{0}, y\right)}(\varphi)\right\|_{W\left(L^{1,1}\right)} \\
& +\sum_{k_{1} \in \mathbf{Z}} \sup _{x \in[0,1]} \sum_{k_{2} \in \mathbf{Z}^{d}} \sup _{y \in[0,1]^{d}} \sup _{\left|y^{\prime}\right| \leq \delta_{0}} \operatorname{osc}_{\left(\delta_{0}, x\right)}(\varphi)\left(x+k_{1}, y+y^{\prime}+k_{2}\right) \\
\leq & \left(2+\left(2\left[\delta_{0}\right]+3\right)^{d}\right)\|\varphi\|_{W\left(L^{1,1}\right)}+\left(2\left[\delta_{0}\right]+3\right)^{d}\left\|\operatorname{osc}_{\left(\delta_{0}, x\right)}(\varphi)\right\|_{W\left(L^{1,1}\right)} .
\end{aligned}
$$


Thus, $\operatorname{osc}_{\delta_{0}}(\varphi) \in W\left(L^{1,1}\right)$. Then for any $\varepsilon>0$, there exists an integer $L_{0}>0$ such that

$$
\begin{aligned}
& \sum \sup _{k_{1} \in \mathbf{Z}} \sum_{x_{1} \in[0,1]} \sup _{\left|k_{2}\right| \geq L_{0}} \operatorname{osc}_{\delta_{0} \in[0,1]^{d}}(\varphi)\left(x_{1}+k_{1}, x_{2}+k_{2}\right)<\frac{\varepsilon}{3},
\end{aligned}
$$

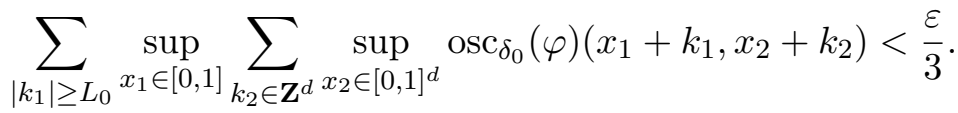

Moreover, since $\varphi$ is continuous, there exists $\delta_{1}>0$ such that

$$
\operatorname{osc}_{\delta}(\varphi)\left(x_{1}+k_{1}, x_{2}+k_{2}\right) \leq \frac{\varepsilon}{3\left(2 L_{0}+1\right)^{d+1}}
$$

for all $\left(x_{1}, x_{2}\right) \in[0,1] \times[0,1]^{d},\left|k_{1}\right| \leq L_{0},\left|k_{2}\right| \leq L_{0}$ and $0<\delta<\delta_{1}$. Then

$$
\sum \sup _{\left|k_{1}\right| \leq L_{0}} \sum_{x_{1} \in[0,1]} \sup _{\left|k_{2}\right| \leq L_{0}} \operatorname{osc}_{\delta}(\varphi)\left(x_{1}+k_{1}, x_{2}+k_{2}\right) \leq \frac{\varepsilon}{3} .
$$

Let $\delta^{\prime}=\min \left\{\delta_{0}, \delta_{1}\right\}$. It follows from (2.4), (2.5) and (2.6) that for any $0<\delta \leq \delta^{\prime}$, one has

$$
\begin{aligned}
\left\|\operatorname{osc}_{\delta}(\varphi)\right\|_{W\left(L^{1,1}\right)} & =\sum_{k_{1} \in \mathbf{Z}} \sup _{x_{1} \in[0,1]} \sum_{k_{2} \in \mathbf{Z}^{d}} \sup _{x_{2} \in[0,1]^{d}} \operatorname{osc}_{\delta}(\varphi)\left(x_{1}+k_{1}, x_{2}+k_{2}\right) \\
& <\frac{\varepsilon}{3}+\frac{\varepsilon}{3}+\frac{\varepsilon}{3}=\varepsilon
\end{aligned}
$$

Thus, $\lim _{\delta \rightarrow 0}\left\|\operatorname{osc}_{\delta}(\varphi)\right\|_{W\left(L^{1,1}\right)}=0$.

Lemma 2.7. Suppose that $\varphi \in W\left(L^{1,1}\right)$ is continuous and $\operatorname{osc}_{\left(\delta_{0}, x\right)}(\varphi) \in W\left(L^{1,1}\right)$ for some $\delta_{0}>0$. If $\psi \in L^{1}\left(\mathbf{R}^{d+1}\right)$ satisfies $\int_{\mathbf{R}} \int_{\mathbf{R}^{d}} \psi(x, y) d x d y=1$, then $\varphi^{a}(x, y)=\varphi(x, y)-$ $\varphi * \psi_{a}^{*}(x, y)$ satisfies

$$
\lim _{a \rightarrow 0}\left\|\varphi^{a}\right\|_{W\left(L^{1,1}\right)}=0,
$$

where $\psi_{a}(\cdot)=\frac{1}{a^{d+1}} \psi(\dot{\bar{a}})$ and $\psi_{a}^{*}(\cdot)=\overline{\psi_{a}(-\cdot)}$.

Proof. Let $\Omega_{\delta_{0}}=\left\{(s, t) \in \mathbf{R} \times \mathbf{R}^{d}:|s| \leq \delta_{0},|t| \leq \delta_{0}\right\}$. Then it follows from the definition of $\varphi^{a}(x, y)$ that

$$
\begin{aligned}
\left|\varphi^{a}(x, y)\right| & \leq\left(\int_{\sqrt{s^{2}+|t|^{2}} \geq \delta_{0}}+\int_{\Omega_{\delta_{0}}}\right)|\varphi(x, y)-\varphi(x+s, y+t)|\left|\psi_{a}(s, t)\right| d s d t \\
& =: I_{1}(x, y)+I_{2}(x, y) .
\end{aligned}
$$

Now, we estimate $\left\|I_{1}\right\|_{W\left(L^{1,1}\right)}$ and $\left\|I_{2}\right\|_{W\left(L^{1,1}\right)}$, respectively. In fact,

$$
\begin{aligned}
& \left\|I_{1}\right\|_{W\left(L^{1,1}\right)} \\
\leq & \sum_{k_{1} \in \mathbf{Z}} \sup _{x \in[0,1]} \sum_{k_{2} \in \mathbf{Z}^{d}} \sup _{y \in[0,1]^{d}} \int_{\sqrt{s^{2}+|t|^{2}} \geq \delta_{0}}\left|\varphi\left(x+k_{1}, y+k_{2}\right)\right|\left|\psi_{a}(s, t)\right| d s d t \\
& +\sum_{k_{1} \in \mathbf{Z}} \sup _{x \in[0,1]} \sum_{k_{2} \in \mathbf{Z}^{d}} \sup _{y \in[0,1]^{d}} \int_{\sqrt{s^{2}+|t|^{2}} \geq \delta_{0}}\left|\varphi\left(x+k_{1}+s, y+k_{2}+t\right) \| \psi_{a}(s, t)\right| d s d t \\
= & : I_{1,1}+I_{1,2} .
\end{aligned}
$$


Since $\psi \in L^{1}\left(\mathbf{R}^{d+1}\right)$, then changing the variables obtains

$$
I_{1,1} \leq\|\varphi\|_{W\left(L^{1,1}\right)} \int_{\sqrt{s^{2}+|t|^{2}} \geq \frac{\delta_{0}}{a}}|\psi(s, t)| d s d t \rightarrow 0 \quad \text { as } a \rightarrow 0 .
$$

Let $\mathbf{Z}_{0}^{d+1}=\left\{\left(\ell_{1}, \ell_{2}\right) \in \mathbf{Z} \times \mathbf{Z}^{d}: \sqrt{\left(\ell_{1}+s\right)^{2}+\left|\ell_{2}+t\right|^{2}} \geq \delta_{0}\right.$ for any $\left.(s, t) \in[0,1] \times[0,1]^{d}\right\}$. Then we have

$$
\begin{aligned}
& I_{1,2} \\
= & \sum_{k_{1} \in \mathbf{Z}} \sup _{x \in[0,1]} \sum_{k_{2} \in \mathbf{Z}^{d}} \sup _{y \in[0,1]^{d}} \int_{\sqrt{s^{2}+|t|^{2}} \geq \delta_{0}}\left|\varphi\left(x+k_{1}+s, y+k_{2}+t\right)\right|\left|\psi_{a}(s, t)\right| d s d t \\
\leq & \sum_{k_{1} \in \mathbf{Z}} \sup _{x \in[0,1]} \sum_{k_{2} \in \mathbf{Z}^{d}} \sup _{y \in[0,1]^{d}} \sum_{\left(\ell_{1}, \ell_{2}\right) \in \mathbf{Z}_{0}^{d+1}} \int_{0}^{1} \int_{[0,1]^{d}}\left|\varphi\left(x+k_{1}+\ell_{1}+s, y+k_{2}+\ell_{2}+t\right)\right| \\
& \times\left|\psi_{a}\left(\ell_{1}+s, \ell_{2}+t\right)\right| d t d s \\
\leq & \sum_{\left(\ell_{1}, \ell_{2}\right) \in \mathbf{Z}_{0}^{d+1}} \int_{0}^{1} \int_{[0,1]^{d}}\left(\sum_{k_{1} \in \mathbf{Z}^{x \in[0,1]}} \sup _{s \in[0,1]} \sum_{k_{2} \in \mathbf{Z}^{d}} \sup _{y \in[0,1]^{d}} \sup _{t \in[0,1]^{d}}\left|\varphi\left(x+k_{1}+s, y+k_{2}+t\right)\right|\right) \\
& \times\left|\psi_{a}\left(\ell_{1}+s, \ell_{2}+t\right)\right| d t d s \\
\leq & 2^{d+1}\|\varphi\|_{W\left(L^{1,1}\right)} \int_{\sqrt{s^{2}+|t|^{2}} \geq \frac{\delta_{0}}{a}}|\psi(s, t)| d s d t \rightarrow 0 \text { as } a \rightarrow 0 .
\end{aligned}
$$

This together with 2.7 and 2.8 proves that $\lim _{a \rightarrow 0}\left\|I_{1}\right\|_{W\left(L^{1,1}\right)}=0$.

Since $\lim _{\delta \rightarrow 0}\left\|\operatorname{osc}_{\delta}(\varphi)\right\|_{W\left(L^{1,1}\right)}=0$, then for any $\epsilon>0$, there exists a $0<\delta_{2}<\delta_{0}$ such that

$$
\left\|\operatorname{osc}_{\delta}(\varphi)\right\|_{W\left(L^{1,1}\right)}<\epsilon, \quad \forall \delta<\delta_{2} .
$$

Now, we begin to estimate $\left\|I_{2}\right\|_{W\left(L^{1,1}\right)}$ :

$$
\begin{aligned}
\left\|I_{2}\right\|_{W\left(L^{1,1}\right)} \leq & \sum_{k_{1} \in \mathbf{Z}} \sup _{x \in[0,1]} \sum_{k_{2} \in \mathbf{Z}^{d}} \sup _{y \in[0,1]^{d}} \int_{\Omega_{\delta_{0}}} \operatorname{osc}_{\max \{|s|,|t|\}}(\varphi)\left(x+k_{1}, y+k_{2}\right)\left|\psi_{a}(s, t)\right| d s d t \\
\leq & \sum_{k_{1} \in \mathbf{Z}} \sup _{x \in[0,1]} \sum_{k_{2} \in \mathbf{Z}^{d}} \sup _{y \in[0,1]^{d}}\left[\int_{\Omega_{\delta_{2} / 2}} \operatorname{osc}_{\delta_{2} / 2}(\varphi)\left(x+k_{1}, y+k_{2}\right)\left|\psi_{a}(s, t)\right| d s d t\right. \\
& \left.\quad+\int_{\sqrt{s^{2}+|t|^{2}} \geq \delta_{2} / 2} \operatorname{osc}_{\delta_{0}}(\varphi)\left(x+k_{1}, y+k_{2}\right)\left|\psi_{a}(s, t)\right| d s d t\right] \\
\leq & \left\|\operatorname{osc}_{\delta_{2} / 2}(\varphi)\right\|_{W\left(L^{1,1}\right)} \int_{\mathbf{R}} \int_{\mathbf{R}^{d}}\left|\psi_{a}(s, t)\right| d t d s \\
& +\left\|\operatorname{osc}_{\delta_{0}}(\varphi)\right\|_{W\left(L^{1,1}\right)} \int_{\sqrt{s^{2}+|t|^{2}} \geq \delta_{2} / 2}\left|\psi_{a}(s, t)\right| d s d t \\
\leq & \epsilon\|\|_{L^{1}}+\left\|\operatorname{osc}_{\delta_{0}}(\varphi)\right\|_{W\left(L^{1,1}\right)} \int_{\sqrt{s^{2}+|t|^{2}} \geq \frac{\delta_{2}}{2 a}}|\psi(s, t)| d s d t .
\end{aligned}
$$

This together with 2.3 obtains $\lim _{a \rightarrow 0}\left\|I_{2}\right\|_{W\left(L^{1,1}\right)}=0$ and the final result holds. 
Lemma 2.8. Let $\varphi, \psi$ and $\varphi^{a}$ be as in Lemma 2.7. Then

$$
\left\|\operatorname{osc}_{\gamma}\left(\varphi^{a}\right)\right\|_{W\left(L^{1,1}\right)} \leq\left(1+2^{d+1}\|\psi\|_{L^{1}}\right)\left\|\operatorname{osc}_{\gamma}(\varphi)\right\|_{W\left(L^{1,1}\right)}
$$

and $\lim _{\gamma \rightarrow 0}\left\|\operatorname{osc}_{\gamma}\left(\varphi^{a}\right)\right\|_{W\left(L^{1,1}\right)}=0$.

Proof. Note that

$$
\begin{aligned}
\operatorname{osc}_{\gamma}\left(\varphi^{a}\right)(x, y) & \leq \operatorname{osc}_{\gamma}(\varphi)(x, y)+\int_{\mathbf{R}} \int_{\mathbf{R}^{d}} \operatorname{osc}_{\gamma}(\varphi)(x+s, y+t)\left|\psi_{a}(s, t)\right| d t d s \\
& =\operatorname{osc}_{\gamma}(\varphi)(x, y)+\operatorname{osc}_{\gamma}(\varphi) *\left|\psi_{a}^{*}\right|(x, y) .
\end{aligned}
$$

Then it follows from Lemma 2.1 that

$$
\left\|\operatorname{osc}_{\gamma}\left(\varphi^{a}\right)\right\|_{W\left(L^{1,1}\right)} \leq\left(1+2^{d+1}\|\psi\|_{L^{1}}\right)\left\|\operatorname{osc}_{\gamma}(\varphi)\right\|_{W\left(L^{1,1}\right)} .
$$

Since $\lim _{\gamma \rightarrow 0}\left\|\operatorname{osc}_{\gamma}(\varphi)\right\|_{W\left(L^{1,1}\right)}=0$, the desired result is proved.

3. Average sampling and reconstruction

In this section, we will discuss the nonuniform average sampling in the multiply generated shift-invariant subspaces $V_{p, q}\left(\Phi_{r}\right)$.

Definition 3.1. A set $\Gamma=\left\{\gamma_{j, k}=\left(x_{j}, y_{k}\right): x_{j} \in \mathbf{R}, y_{k} \in \mathbf{R}^{d}, j \in \mathbb{J}_{1}, k \in \mathbb{J}_{2}\right\}$ is called a $\gamma_{0}$-dense set if

$$
\mathbf{R}^{d+1}=\bigcup_{j, k} B_{\gamma}\left(x_{j}, y_{k}\right), \quad \forall \gamma>\gamma_{0} .
$$

Here, $B_{\gamma}\left(x_{j}, y_{k}\right)$ is an open ball in $\mathbf{R}^{d+1}$ with center $\left(x_{j}, y_{k}\right)$ and radius $\gamma, \mathbb{J}_{1}$ and $\mathbb{J}_{2}$ are countable index sets.

Definition 3.2. The family $U=\left\{u_{j, k}(x, y)\right\}_{j \in \mathbb{J}_{1}, k \in \mathbb{J}_{2}}$ is a bounded uniform partition of unity (BUPU) associated with $\Gamma$ if

(i) $0 \leq u_{j, k}(x, y) \leq 1$ for all $(x, y) \in \mathbf{R} \times \mathbf{R}^{d}, j \in \mathbb{J}_{1}, k \in \mathbb{J}_{2}$;

(ii) $u_{j, k}(x, y)$ is supported in $B_{\gamma}\left(x_{j}, y_{k}\right)$ for every $j \in \mathbb{J}_{1}, k \in \mathbb{J}_{2}$;

(iii) $\sum_{j \in \mathbb{J}_{1}} \sum_{k \in \mathbb{J}_{2}} u_{j, k}(x, y)=1$ for all $(x, y) \in \mathbf{R} \times \mathbf{R}^{d}$.

Obviously, $u_{j, k}(x, y)=\frac{\chi_{B\left(x_{j}, \gamma / \sqrt{2}\right)}(x)}{\sum_{j^{\prime} \in \mathbb{J}_{1}} \chi_{B\left(x_{j^{\prime}}, \gamma / \sqrt{2}\right)}(x)} \cdot \frac{\chi_{B\left(y_{k}, \gamma / \sqrt{2}\right)}(y)}{\sum_{k^{\prime} \in \mathbb{J}_{2}} \chi_{B\left(y_{k^{\prime}}, \gamma / \sqrt{2}\right)}(y)}, j \in \mathbb{J}_{1}, k \in \mathbb{J}_{2}$ is an example of bounded uniform partition of unity. Here, $B\left(y_{k}, \gamma / \sqrt{2}\right)$ denotes a ball in $\mathbf{R}^{d}$ with radius $\gamma / \sqrt{2}$ centered at $y_{k}$.

If $f \in V_{p, q}\left(\Phi_{r}\right)$, define the interpolation operator

$$
Q_{\Gamma} f=\sum_{j \in \mathbb{J}_{1}} \sum_{k \in \mathbb{J}_{2}} f\left(x_{j}, y_{k}\right) u_{j, k} .
$$

Then we have the following conclusion. 
Lemma 3.3. Suppose that $\left\{\varphi_{i}\right\}_{1 \leq i \leq r}$ satisfies the assumptions (A2) and (A3), then for any $\gamma>0$ and $f=\sum_{i=1}^{r} \sum_{k_{1} \in \mathbf{Z}} \sum_{k_{2} \in \mathbf{Z}^{d}} c_{i}\left(k_{1}, k_{2}\right) \varphi_{i}\left(\cdot-k_{1}, \cdot-k_{2}\right) \in V_{p, q}\left(\Phi_{r}\right)$, one has

$$
\left\|Q_{\Gamma} f\right\|_{L^{p, q}} \leq\|c\|_{\ell^{p, q}} \max _{1 \leq i \leq r}\left\{\left\|\varphi_{i}\right\|_{W\left(L^{1,1}\right)}+\left\|\operatorname{osc}_{\gamma}\left(\varphi_{i}\right)\right\|_{W\left(L^{1,1}\right)}\right\} .
$$

Here, $\|c\|_{\ell^{p, q}}=\sum_{i=1}^{r}\left\|c_{i}\right\|_{\ell^{p, q}}$ for $c=\left(c_{1}, c_{2}, \ldots, c_{r}\right)^{T} \in\left(\ell^{p, q}\left(\mathbf{Z} \times \mathbf{Z}^{d}\right)\right)^{r}$.

Proof. By Lemma 2.3, one has

$$
\begin{aligned}
\left\|f-Q_{\Gamma} f\right\|_{L^{p, q}} & =\left\|f(x, y) \sum_{j \in \mathbb{J}_{1}} \sum_{k \in \mathbb{J}_{2}} u_{j, k}(x, y)-\sum_{j \in \mathbb{J}_{1}} \sum_{k \in \mathbb{J}_{2}} f\left(x_{j}, y_{k}\right) u_{j, k}(x, y)\right\|_{L^{p, q}} \\
& \leq\left\|\sum_{j \in \mathbb{J}_{1}} \sum_{k \in \mathbb{J}_{2}}\left|f(x, y)-f\left(x_{j}, y_{k}\right)\right| u_{j, k}(x, y)\right\|_{L^{p, q}} \\
& \leq\left\|\sum_{j \in \mathbb{J}_{1}} \sum_{k \in \mathbb{J}_{2}} \operatorname{osc}_{\gamma}(f)(x, y) u_{j, k}(x, y)\right\|_{L^{p, q}} \\
& =\left\|\operatorname{osc}_{\gamma}(f)\right\|_{L^{p, q}} \leq \sum_{i=1}^{r}\left\|c_{i}\right\|_{\ell^{p, q}}\left\|\operatorname{osc}_{\gamma}\left(\varphi_{i}\right)\right\|_{W\left(L^{1,1}\right)}
\end{aligned}
$$

Moreover, we can obtain

$$
\begin{aligned}
\left\|Q_{\Gamma} f\right\|_{L^{p, q}} & \leq\left\|f-Q_{\Gamma} f\right\|_{L^{p, q}}+\|f\|_{L^{p, q}} \\
& \leq \sum_{i=1}^{r}\left\|c_{i}\right\|_{\ell^{p, q}}\left\|\operatorname{osc}_{\gamma}\left(\varphi_{i}\right)\right\|_{W\left(L^{1,1}\right)}+\sum_{i=1}^{r}\left\|c_{i}\right\|_{\ell^{p, q}}\left\|\varphi_{i}\right\|_{W\left(L^{1,1}\right)} .
\end{aligned}
$$

This completes the proof.

\subsection{The first average sampling}

The first average sampling scheme is convolution sampling which is defined as

$$
\left\langle f(\cdot), \psi_{a}\left(\cdot-\left(x_{j}, y_{k}\right)\right)\right\rangle=f * \psi_{a}^{*}\left(x_{j}, y_{k}\right), \quad j \in \mathbb{J}_{1}, k \in \mathbb{J}_{2},
$$

where $\psi \in L^{1}\left(\mathbf{R}^{d+1}\right)$ satisfies $\int_{\mathbf{R}} \int_{\mathbf{R}^{d}} \psi(x, y) d x d y=1$.

Based on the average samples obtained in (3.2), define the prereconstruction operator

$$
A_{\Gamma, a} f=\sum_{j \in \mathbb{J}_{1}} \sum_{k \in \mathbb{J}_{2}} f * \psi_{a}^{*}\left(x_{j}, y_{k}\right) u_{j, k} .
$$

Lemma 3.4. Let $P$ be a bounded projection from $L^{p, q}\left(\mathbf{R} \times \mathbf{R}^{d}\right)$ onto $V_{p, q}\left(\Phi_{r}\right)$. Suppose that $\left\{\varphi_{i}\right\}_{1 \leq i \leq r}$ satisfies the assumptions (A1)-(A3), then there exist $\gamma_{0}>0$ and $a_{0}>0$ such that for every $\gamma$-dense set $\Gamma$ with $\gamma \leq \gamma_{0}$ and for every positive $a \leq a_{0}$, the operator $I-P A_{\Gamma, a}$ is a contraction on $V_{p, q}\left(\Phi_{r}\right)$. 
Proof. Suppose that $f=\sum_{i=1}^{r} \sum_{k_{1} \in \mathbf{Z}} \sum_{k_{2} \in \mathbf{Z}^{d}} c_{i}\left(k_{1}, k_{2}\right) \varphi_{i}\left(\cdot-k_{1}, \cdot-k_{2}\right) \in V_{p, q}\left(\Phi_{r}\right)$. Since $P f=f$, then

$$
\begin{aligned}
\left\|f-P A_{\Gamma, a} f\right\|_{L^{p, q}} & =\left\|f-P Q_{\Gamma} f+P Q_{\Gamma} f-P A_{\Gamma, a} f\right\|_{L^{p, q}} \\
& \leq\left\|f-P Q_{\Gamma} f\right\|_{L^{p, q}}+\left\|P Q_{\Gamma} f-P A_{\Gamma, a} f\right\|_{L^{p, q}} \\
& \leq\|P\|_{\text {op }}\left(\left\|f-Q_{\Gamma} f\right\|_{L^{p, q}}+\left\|Q_{\Gamma} f-A_{\Gamma, a} f\right\|_{L^{p, q}}\right),
\end{aligned}
$$

where $\|P\|_{\text {op }}$ denotes the operator norm of $P$. Moreover, it follows from (1.1) and (3.1) that

$$
\begin{aligned}
\left\|f-Q_{\Gamma} f\right\|_{L^{p, q}} & \leq \sum_{i=1}^{r}\left\|c_{i}\right\|_{\ell^{p, q}}\left\|\operatorname{osc}_{\gamma}\left(\varphi_{i}\right)\right\|_{W\left(L^{1,1}\right)} \\
& \leq B\|f\|_{L^{p, q}} \max _{1 \leq i \leq r}\left\|\operatorname{osc}_{\gamma}\left(\varphi_{i}\right)\right\|_{W\left(L^{1,1}\right)}
\end{aligned}
$$

Let $\varphi_{i}^{a}(x, y)=\varphi_{i}(x, y)-\varphi_{i} * \psi_{a}^{*}(x, y), 1 \leq i \leq r$. Note that

$$
Q_{\Gamma} f-A_{\Gamma, a} f=Q_{\Gamma}\left(\sum_{i=1}^{r} \sum_{k_{1} \in \mathbf{Z}} \sum_{k_{2} \in \mathbf{Z}^{d}} c_{i}\left(k_{1}, k_{2}\right) \varphi_{i}^{a}\left(\cdot-k_{1}, \cdot-k_{2}\right)\right) .
$$

This together with Lemma 3.3 and 1.1 obtains

$$
\begin{aligned}
\left\|Q_{\Gamma} f-A_{\Gamma, a} f\right\|_{L^{p, q}} & =\left\|\sum_{i=1}^{r} Q_{\Gamma}\left(\sum_{k_{1} \in \mathbf{Z}} \sum_{k_{2} \in \mathbf{Z}^{d}} c_{i}\left(k_{1}, k_{2}\right) \varphi_{i}^{a}\left(\cdot-k_{1}, \cdot-k_{2}\right)\right)\right\|_{L^{p, q}} \\
& \leq \sum_{i=1}^{r}\left\|c_{i}\right\|_{\ell^{p, q}} \max _{1 \leq i \leq r}\left\{\left\|\varphi_{i}^{a}\right\|_{W\left(L^{1,1}\right)}+\left\|\operatorname{osc}_{\gamma}\left(\varphi_{i}^{a}\right)\right\|_{W\left(L^{1,1}\right)}\right\} \\
& \leq B\|f\|_{L^{p, q}} \max _{1 \leq i \leq r}\left\{\left\|\varphi_{i}^{a}\right\|_{W\left(L^{1,1}\right)}+\left\|\operatorname{osc}_{\gamma}\left(\varphi_{i}^{a}\right)\right\|_{W\left(L^{1,1}\right)}\right\} .
\end{aligned}
$$

This together with 3.3 and 3.4 obtains

$$
\begin{aligned}
& \left\|f-P A_{\Gamma, a} f\right\|_{L^{p, q}} \\
\leq & B\|P\|_{\text {op }}\left(\max _{1 \leq i \leq r}\left\|\operatorname{osc}_{\gamma}\left(\varphi_{i}\right)\right\|_{W\left(L^{1,1}\right)}+\max _{1 \leq i \leq r}\left\{\left\|\varphi_{i}^{a}\right\|_{W\left(L^{1,1}\right)}+\left\|\operatorname{osc}_{\gamma}\left(\varphi_{i}^{a}\right)\right\|_{W\left(L^{1,1}\right)}\right\}\right)\|f\|_{L^{p, q}} .
\end{aligned}
$$

Therefore, it follows from Lemmas 2.6, 2.7 and 2.8 that we can choose positive numbers $\gamma_{0}$ and $a_{0}$ such that for any $\gamma \leq \gamma_{0}$ and $a \leq a_{0}$, one has

$$
B\|P\|_{\text {op }}\left(\max _{1 \leq i \leq r}\left\|\operatorname{osc}_{\gamma}\left(\varphi_{i}\right)\right\|_{W\left(L^{1,1}\right)}+\max _{1 \leq i \leq r}\left\{\left\|\varphi_{i}^{a}\right\|_{W\left(L^{1,1}\right)}+\left\|\operatorname{osc}_{\gamma}\left(\varphi_{i}^{a}\right)\right\|_{W\left(L^{1,1}\right)}\right\}\right)<1,
$$

which means that $I-P A_{\Gamma, a}$ is a contraction on $V_{p, q}\left(\Phi_{r}\right)$. 
Theorem 3.5. Let $P,\left\{\varphi_{i}\right\}_{1 \leq i \leq r}, \Gamma$ and $A_{\Gamma, a}$ be as in Lemma 3.4. Then any signal $f \in V_{p, q}\left(\Phi_{r}\right)$ can be recovered from its average samples $\left\{\left\langle f(\cdot), \psi_{a}\left(\cdot-\left(x_{j}, y_{k}\right)\right)\right\rangle\right\}_{j \in \mathbb{J}_{1}, k \in \mathbb{J}_{2}}$ by the iterative approximation projection algorithm

$$
f_{1}=P A_{\Gamma, a} f \quad \text { and } \quad f_{n+1}=P A_{\Gamma, a}\left(f-f_{n}\right)+f_{n} .
$$

Here, $f_{n}$ uniformly converges to $f$, and also in $W\left(L^{p, q}\right)$ and $L^{p, q}$-norms just as

$$
\left\|f-f_{n}\right\|_{L^{p, q}} \leq\left\|f-f_{n}\right\|_{W\left(L^{p, q}\right)} \leq M \alpha_{1}^{n}\|f\|_{L^{p, q}}
$$

for some $\alpha_{1}=\alpha_{1}(\gamma, a, \Phi, \psi)<1$ and $M<\infty$.

Proof. Let $e_{n}=f-f_{n}$. Then

$$
e_{n+1}=f-f_{n+1}=\left(I-P A_{\Gamma, a}\right) e_{n}
$$

Set

$$
\alpha_{1}=B\|P\|_{\text {op }}\left(\max _{1 \leq i \leq r}\left\|\operatorname{osc}_{\gamma}\left(\varphi_{i}\right)\right\|_{W\left(L^{1,1}\right)}+\max _{1 \leq i \leq r}\left\{\left\|\varphi_{i}^{a}\right\|_{W\left(L^{1,1}\right)}+\left\|\operatorname{osc}_{\gamma}\left(\varphi_{i}^{a}\right)\right\|_{W\left(L^{1,1}\right)}\right\}\right) .
$$

By Lemma 3.4, we may choose $\gamma>0$ and $a>0$ small enough such that $\alpha_{1}<1$ and

$$
\left\|e_{n+1}\right\|_{L^{p, q}} \leq \alpha_{1}\left\|e_{n}\right\|_{L^{p, q}} \leq \alpha_{1}^{n+1}\|f\|_{L^{p, q}}
$$

Thus, it follows from Lemma 2.4 that 3.5 holds with $M=B\left(\max _{1 \leq i \leq r}\left\|\varphi_{i}\right\|_{W\left(L^{1,1}\right)}\right)$.

\subsection{The second average sampling}

The second average sampling scheme is

$$
\left\langle f, \psi_{j, k}\right\rangle=\int_{\mathbf{R}} \int_{\mathbf{R}^{d}} f(x, y) \psi_{j, k}(x, y) d x d y, \quad j \in \mathbb{J}_{1}, k \in \mathbb{J}_{2},
$$

where the average sampling functionals $\left\{\psi_{j, k}: j \in \mathbb{J}_{1}, k \in \mathbb{J}_{2}\right\}$ satisfy

(i) $\int_{\mathbf{R}} \int_{\mathbf{R}^{d}} \psi_{j, k}(x, y) d x d y=1$ for all $j \in \mathbb{J}_{1}, k \in \mathbb{J}_{2}$;

(ii) There exists an $R>0$ such that $\int_{\mathbf{R}} \int_{\mathbf{R}^{d}}\left|\psi_{j, k}(x, y)\right| d x d y \leq R$ for all $j \in \mathbb{J}_{1}, k \in \mathbb{J}_{2}$;

(iii) $\operatorname{supp} \psi_{j, k} \subset B_{a}\left(x_{j}, y_{k}\right)$ for some $a>0$.

Define the quasi-reconstruction operator

$$
A_{\Gamma} f=\sum_{j \in \mathbb{J}_{1}} \sum_{k \in \mathbb{J}_{2}}\left\langle f, \psi_{j, k}\right\rangle u_{j, k}
$$

Then we have the following conclusion. 
Theorem 3.6. Let $P$ be a bounded projection from $L^{p, q}\left(\mathbf{R} \times \mathbf{R}^{d}\right)$ onto $V_{p, q}\left(\Phi_{r}\right)$. Suppose that $\left\{\varphi_{i}\right\}_{1 \leq i \leq r}$ satisfies the assumptions (A1)-(A3), then there exist $\gamma>0$ and $a>0$ such that any $f \in V_{p, q}\left(\Phi_{r}\right)$ can be recovered from its average samples $\left\{\left\langle f, \psi_{j, k}\right\rangle\right\}_{j \in \mathbb{J}_{1}, k \in \mathbb{J}_{2}}$ by the iterative algorithm

$$
f_{1}=P A_{\Gamma} f \quad \text { and } \quad f_{n+1}=P A_{\Gamma}\left(f-f_{n}\right)+f_{n},
$$

which converges uniformly and also satisfies

$$
\left\|f-f_{n}\right\|_{L^{p, q}} \leq\left\|f-f_{n}\right\|_{W\left(L^{p, q}\right)} \leq M \alpha_{2}^{n}\|f\|_{L^{p, q}}
$$

for some $\alpha_{2}=\alpha_{2}(\gamma, a, \Phi, R)<1$.

Proof. Let $f=\sum_{i=1}^{r} \sum_{k_{1} \in \mathbf{Z}} \sum_{k_{2} \in \mathbf{Z}^{d}} c_{i}\left(k_{1}, k_{2}\right) \varphi_{i}\left(\cdot-k_{1}, \cdot-k_{2}\right) \in V_{p, q}\left(\Phi_{r}\right)$. Then

$$
\begin{aligned}
\left\|f-P A_{\Gamma} f\right\|_{L^{p, q}} & =\left\|f-P Q_{\Gamma} f+P Q_{\Gamma} f-P A_{\Gamma} f\right\|_{L^{p, q}} \\
& \leq\|P\|_{\text {op }}\left(\left\|f-Q_{\Gamma} f\right\|_{L^{p, q}}+\left\|Q_{\Gamma} f-A_{\Gamma} f\right\|_{L^{p, q}}\right) .
\end{aligned}
$$

Note that

$$
\operatorname{osc}_{a}(f) \leq \sum_{i=1}^{r} \sum_{k_{1} \in \mathbf{Z}} \sum_{k_{2} \in \mathbf{Z}^{d}}\left|c_{i}\left(k_{1}, k_{2}\right)\right| \operatorname{osc}_{a}\left(\varphi_{i}\right)\left(\cdot-k_{1}, \cdot-k_{2}\right) .
$$

Then, it follows from Lemma 3.3 that

$$
\begin{aligned}
& \left\|Q_{\Gamma} f-A_{\Gamma} f\right\|_{L^{p, q}} \\
\leq & \left\|\sum _ { j \in \mathbb { J } _ { 1 } } \sum _ { k \in \mathbb { J } _ { 2 } } \int _ { \mathbf { R } } \int _ { \mathbf { R } ^ { d } } \left|f\left(x_{j}, y_{k}\right)-f(s, t)\left\|\psi_{j, k}(s, t) \mid d s d t u_{j, k}(x, y)\right\|_{L^{p, q}}\right.\right. \\
\leq & R\left\|\sum_{j \in \mathbb{J}_{1}} \sum_{k \in \mathbb{J}_{2}} \operatorname{osc}_{a}(f)\left(x_{j}, y_{k}\right) u_{j, k}(x, y)\right\|_{L^{p, q}} \\
\leq & R\|c\|_{\ell^{p, q}} \max _{1 \leq i \leq r}\left\{\left\|\operatorname{osc}_{a}\left(\varphi_{i}\right)\right\|_{W\left(L^{1,1}\right)}+\left\|\operatorname{osc}_{\gamma}\left(\operatorname{osc}_{a}\left(\varphi_{i}\right)\right)\right\|_{W\left(L^{1,1}\right)}\right\} \\
\leq & R B\|f\|_{L^{p, q}} \max _{1 \leq i \leq r}\left\{\left\|\operatorname{osc}_{a}\left(\varphi_{i}\right)\right\|_{W\left(L^{1,1}\right)}+\left\|\operatorname{osc}_{\gamma}\left(\operatorname{osc}_{a}\left(\varphi_{i}\right)\right)\right\|_{W\left(L^{1,1}\right)}\right\} \\
\leq & R B\|f\|_{L^{p, q}} \max _{1 \leq i \leq r}\left\{2\left\|\operatorname{osc}_{a}\left(\varphi_{i}\right)\right\|_{W\left(L^{1,1}\right)}+\left\|\operatorname{osc}_{\gamma}\left(\varphi_{i}\right)\right\|_{W\left(L^{1,1}\right)}+\left\|\operatorname{osc}_{a+\gamma}\left(\varphi_{i}\right)\right\|_{W\left(L^{1,1}\right)}\right\} .
\end{aligned}
$$

This together with 3.7 obtains

$$
\begin{aligned}
& \left\|f-P A_{\Gamma} f\right\|_{L^{p, q}} \\
& \leq B\|P\|_{\text {op }}\left(\max _{1 \leq i \leq r}\left\|\operatorname{osc}_{\gamma}\left(\varphi_{i}\right)\right\|_{W\left(L^{1,1}\right)}\right. \\
& \left.\quad+R \max _{1 \leq i \leq r}\left\{2\left\|\operatorname{osc}_{a}\left(\varphi_{i}\right)\right\|_{W\left(L^{1,1}\right)}+\left\|\operatorname{osc}_{\gamma}\left(\varphi_{i}\right)\right\|_{W\left(L^{1,1}\right)}+\left\|\operatorname{osc}_{a+\gamma}\left(\varphi_{i}\right)\right\|_{W\left(L^{1,1}\right)}\right\}\right)\|f\|_{L^{p, q}} .
\end{aligned}
$$


Take $\gamma>0$ and $a>0$ small enough such that

$$
\begin{aligned}
& \alpha_{2}=B\|P\|_{\text {op }}\left(\max _{1 \leq i \leq r}\left\|\operatorname{osc}_{\gamma}\left(\varphi_{i}\right)\right\|_{W\left(L^{1,1}\right)}\right. \\
& \left.\quad+R \max _{1 \leq i \leq r}\left\{2\left\|\operatorname{osc}_{a}\left(\varphi_{i}\right)\right\|_{W\left(L^{1,1}\right)}+\left\|\operatorname{osc}_{\gamma}\left(\varphi_{i}\right)\right\|_{W\left(L^{1,1}\right)}+\left\|\operatorname{osc}_{a+\gamma}\left(\varphi_{i}\right)\right\|_{W\left(L^{1,1}\right)}\right\}\right) \\
& \quad<1 .
\end{aligned}
$$

Then the operator $I-P A_{\Gamma}$ is a contraction on $V_{p, q}\left(\Phi_{r}\right)$ and the remained proof is similar to that of Theorem 3.5 .

\section{Convergence rate}

In this section, we estimate the convergence rate $\alpha_{1}$ in Theorem 3.5 and $\alpha_{2}$ in Theorem 3.6 when $\Phi_{r}$ and the average sampling functionals satisfy some additional regularity conditions.

Theorem 4.1. Suppose that $\left\{\varphi_{i}\right\}_{1 \leq i \leq r}$ and $\psi$ satisfy the conditions of Theorem 3.5, and that $\left|\nabla \varphi_{i}\right| \in W\left(L^{1}\right)\left(\mathbf{R}^{d+1}\right)$ for every $i=1, \ldots, r$ and $\|\psi\|_{1, \eta}:=\int_{\mathbf{R}} \int_{\mathbf{R}^{d}}|\psi(s, t) \|(s, t)|^{\eta} d s d t$ $<\infty$ for some $0<\eta \leq 1$. Then the convergence rate $\alpha_{1}$ in Theorem 3.5 satisfies

$$
\begin{aligned}
\alpha_{1} \leq & B\|P\|_{\text {op }}\left(\sqrt{2} \gamma\left(2+2^{d+1}\|\psi\|_{L^{1}}\right)(3+2[\gamma])^{d+1} \max _{1 \leq i \leq r}\left\|\left|\nabla \varphi_{i}\right|\right\|_{W\left(L^{1}\right)}+a^{\eta}\|\psi\|_{1, \eta}\right. \\
& \left.\times \max _{1 \leq i \leq r}\left(\left(1+2^{d+1}\right) \delta_{0}^{-\eta}\left\|\varphi_{i}\right\|_{W\left(L^{1,1}\right)}+\sqrt{2} \delta_{0}^{1-\eta}\left(3+2\left[\delta_{0}\right]\right)^{d+1}\left\|\left|\nabla \varphi_{i}\right|\right\|_{W\left(L^{1}\right)}\right)\right) .
\end{aligned}
$$

Proof. Note that

$$
\begin{aligned}
\left|\varphi\left(x_{1}+y_{1}, x_{2}+y_{2}\right)-\varphi\left(x_{1}, x_{2}\right)\right| & =\left|\int_{0}^{1}\left(y_{1}, y_{2}\right) \cdot \nabla \varphi\left(x_{1}+s y_{1}, x_{2}+s y_{2}\right) d s\right| \\
& \leq \int_{0}^{1}\left|\left(y_{1}, y_{2}\right)\right||\nabla \varphi|\left(x_{1}+s y_{1}, x_{2}+s y_{2}\right) d s \\
& \leq\left|\left(y_{1}, y_{2}\right)\right| \sup _{\left|t_{1}\right| \leq\left|y_{1}\right|,\left|t_{2}\right| \leq\left|y_{2}\right|}|\nabla \varphi|\left(x_{1}+t_{1}, x_{2}+t_{2}\right),
\end{aligned}
$$

where $\cdot$ in the first equality denotes the vector inner product. Therefore, we can obtain

$$
\begin{aligned}
\operatorname{osc}_{\gamma}(\varphi)\left(x_{1}, x_{2}\right) & =\sup _{\left|y_{1}\right| \leq \gamma,\left|y_{2}\right| \leq \gamma}\left|\varphi\left(x_{1}+y_{1}, x_{2}+y_{2}\right)-\varphi\left(x_{1}, x_{2}\right)\right| \\
& \leq \sqrt{2} \gamma \sup _{\left|t_{1}\right| \leq \gamma,\left|t_{2}\right| \leq \gamma}|\nabla \varphi|\left(x_{1}+t_{1}, x_{2}+t_{2}\right) .
\end{aligned}
$$


Moreover, one has

$$
\begin{aligned}
& \left\|\operatorname{osc}_{\gamma}(\varphi)\right\|_{W\left(L^{1,1}\right)} \\
= & \sum_{k_{1} \in \mathbf{Z}} \sup _{x_{1} \in[0,1]} \sum_{k_{2} \in \mathbf{Z}^{d}} \sup _{x_{2} \in[0,1]^{d}}\left|\operatorname{osc}_{\gamma}(\varphi)\left(x_{1}+k_{1}, x_{2}+k_{2}\right)\right| \\
\leq & \sqrt{2} \gamma \sum_{k_{1} \in \mathbf{Z}} \sup _{x_{1} \in[0,1]} \sum_{k_{2} \in \mathbf{Z}^{d}} \sup _{x_{2} \in[0,1]^{d}} \sup _{\left|t_{1}\right| \leq \gamma,\left|t_{2}\right| \leq \gamma}|\nabla \varphi|\left(x_{1}+t_{1}+k_{1}, x_{2}+t_{2}+k_{2}\right) \\
\leq & \sqrt{2} \gamma \sum_{k_{1} \in \mathbf{Z}} \sum_{k_{2} \in \mathbf{Z}^{d}} \sup _{y_{1} \in[-\gamma, 1+\gamma]} \sup _{y_{2} \in[-\gamma, 1+\gamma]^{d}}|\nabla \varphi|\left(y_{1}+k_{1}, y_{2}+k_{2}\right) \\
\leq & \sqrt{2} \gamma(3+2[\gamma])^{d+1}|| \nabla \varphi \mid \|_{W\left(L^{1}\right)^{*}}
\end{aligned}
$$

Next, we estimate the $W\left(L^{1,1}\right)$-norm of $\varphi^{a}=\varphi-\varphi * \psi_{a}^{*}$. Following the proof of Lemma 2.7 . we have

$$
\left\|I_{1}\right\|_{W\left(L^{1,1}\right)} \leq\left(1+2^{d+1}\right) a^{\eta} \delta_{0}^{-\eta}\|\varphi\|_{W\left(L^{1,1}\right)}\|\psi\|_{1, \eta} .
$$

Moreover, one has

$$
\begin{aligned}
I_{2}(x, y) & \leq \int_{\Omega_{\delta_{0}}}|(s, t)| \sup _{\left|s_{1}\right| \leq \delta_{0},\left|t_{1}\right| \leq \delta_{0}}|\nabla \varphi|\left(x+s_{1}, y+t_{1}\right)\left|\psi_{a}(s, t)\right| d s d t \\
& \leq \sqrt{2} a^{\eta} \delta_{0}^{1-\eta}\|\psi\|_{1, \eta} \sup _{\left|s_{1}\right| \leq \delta_{0},\left|t_{1}\right| \leq \delta_{0}}|\nabla \varphi|\left(x+s_{1}, y+t_{1}\right) .
\end{aligned}
$$

This together with 4.3 obtains

$$
\begin{aligned}
\left\|\varphi^{a}\right\|_{W\left(L^{1,1}\right)} \leq & \left(1+2^{d+1}\right) a^{\eta} \delta_{0}^{-\eta}\|\varphi\|_{W\left(L^{1,1}\right)}\|\psi\|_{1, \eta} \\
& +\sqrt{2} a^{\eta} \delta_{0}^{1-\eta}\left(3+2\left[\delta_{0}\right]\right)^{d+1}\|\mid \nabla \varphi\|_{W\left(L^{1}\right)}\|\psi\|_{1, \eta} .
\end{aligned}
$$

Finally, it follows from Lemma 2.8 and $(3.6)$ that the desired result (4.1) holds.

Theorem 4.2. Suppose that $\left\{\varphi_{i}\right\}_{1 \leq i \leq r}$ and $\left\{\psi_{j, k}: j \in \mathbb{J}_{1}, k \in \mathbb{J}_{2}\right\}$ satisfy the conditions of Theorem 3.6, and that $\left|\nabla \varphi_{i}\right| \in W\left(L^{1}\right)\left(\mathbf{R}^{d+1}\right)$ for every $i=1, \ldots, r$. Then the convergence rate $\alpha_{2}$ in Theorem 3.6 satisfies

$$
\begin{aligned}
\alpha_{2} \leq & \sqrt{2} B\|P\|_{\text {op }}\left(\gamma(1+R)(3+2[\gamma])^{d+1}+2 a R(3+2[a])^{d+1}+(a+\gamma) R(3+2[a+\gamma])^{d+1}\right) \\
& \times \max _{1 \leq i \leq r}\left\|\mid \nabla \varphi_{i}\right\|_{W\left(L^{1}\right)} .
\end{aligned}
$$

Proof. The proof of Theorem 4.2 is a direct consequence of (3.8) and (4.2).

Remark 4.3. Note that the convergence rates $\alpha_{1}$ in Theorem 3.5 and $\alpha_{2}$ in Theorem 3.6 are given as

$$
\alpha_{1}=B\|P\|_{\text {op }}\left(\max _{1 \leq i \leq r}\left\|\operatorname{osc}_{\gamma}\left(\varphi_{i}\right)\right\|_{W\left(L^{1,1}\right)}+\max _{1 \leq i \leq r}\left\{\left\|\varphi_{i}^{a}\right\|_{W\left(L^{1,1}\right)}+\left\|\operatorname{osc}_{\gamma}\left(\varphi_{i}^{a}\right)\right\|_{W\left(L^{1,1}\right)}\right\}\right)
$$


and

$$
\begin{aligned}
\alpha_{2}=B & \|P\|_{\text {op }}\left(\max _{1 \leq i \leq r}\left\|\operatorname{osc}_{\gamma}\left(\varphi_{i}\right)\right\|_{W\left(L^{1,1}\right)}\right. \\
& \left.+R \max _{1 \leq i \leq r}\left\{2\left\|\operatorname{osc}_{a}\left(\varphi_{i}\right)\right\|_{W\left(L^{1,1}\right)}+\left\|\operatorname{osc}_{\gamma}\left(\varphi_{i}\right)\right\|_{W\left(L^{1,1}\right)}+\left\|\operatorname{osc}_{a+\gamma}\left(\varphi_{i}\right)\right\|_{W\left(L^{1,1}\right)}\right\}\right) .
\end{aligned}
$$

Although we know from Lemmas 2.6, 2.7 and 2.8 that both $\alpha_{1}<1$ and $\alpha_{2}<1$ can be achieved by taking $\gamma>0$ and $a>0$ small enough, we do not have the expression of the dependence of $\alpha_{1}$ and $\alpha_{2}$ on $\gamma$ and $a$. Under suitable conditions, Theorems 4.1 and 4.2 obtain explicit bounds for $\alpha_{1}$ and $\alpha_{2}$, respectively, which allow us to find sufficient conditions for density $\gamma$ and size $a$ such that $\alpha_{1}$ and $\alpha_{2}$ can be arbitrary small.

\section{Acknowledgments}

The project is partially supported by the National Natural Science Foundation of China (No. 11661024) and the Guangxi Natural Science Foundation (Nos. 2020GXNSFAA159076, 2019GXNSFFA245012, 2017GXNSFAA198194), Guangxi Key Laboratory of Cryptography and Information Security (No. GCIS201925), Guangxi Colleges and Universities Key Laboratory of Data Analysis and Computation.

\section{References}

[1] A. Aldroubi and H. Feichtinger, Exact iterative reconstruction algorithm for multivariate irregularly sampled functions in spline-like spaces: The $L^{p}$ theory, Proc. Amer. Math. Soc. 126 (1998), no. 9, 2677-2686.

[2] A. Aldroubi and K. Gröcheng, Nonuniform sampling and reconstruction in shiftinvariant spaces, SIAM Rev. 43 (2001), no. 4, 585-620.

[3] A. Aldroubi, Q. Sun and W.-S. Tang, Nonuniform average sampling and reconstruction in multiply generated shift-invariant spaces, Constr. Approx. 20 (2004), no. 2, $173-189$.

[4] A. Benedek and R. Panzone, The space $L^{p}$ with mixed norm, Duke Math. J. 28 (1961), no. 3, 301-324.

[5] G. Cleanthous and A. G. Georgiadis, Mixed-norm $\alpha$-modulation spaces, Trans. Amer. Math. Soc. 373 (2020), no. 5, 3323-3356.

[6] G. Cleanthous, A. G. Georgiadis and M. Nielsen, Molecular decomposition of anisotropic homogeneous mixed-norm spaces with applications to the boundedness of operators, Appl. Comput. Harmon. Anal. 47 (2019), no. 2, 447-480. 
[7] _ Fourier multipliers on anisotropic mixed-norm spaces of distributions, Math. Scand. 124 (2019), no. 2, 289-304.

[8] J. L. Rubio de Francia, F. J. Ruiz and J.-L. Torrea, Calderón-Zygmund theory for operator-valued kernels, Adv. in Math. 62 (1986), no. 1, 7-48.

[9] H. Führ and J. Xian, Relevant sampling in finitely generated shift-invariant spaces, J. Approx. Theory 240 (2019), 1-15.

[10] A. G. Georgiadis, J. Johnsen and M. Nielsen, Wavelet transforms for homogeneous mixed-norm Triebel-Lizorkin spaces, Monatsh. Math. 183 (2017), no. 4, 587-624.

[11] J. Hart, R. H. Torres and X. Wu, Smoothing properties of bilinear operators and Leibniz-type rules in Lebesgue and mixed Lebesgue spaces, Trans. Amer. Math. Soc. 370 (2018), no. 12, 8581-8612.

[12] L. Hörmander, Estimates for translation invariant operators in $L^{p}$ spaces, Acta Math. 104 (1960), 93-140.

[13] L. Huang, J. Liu, D. Yang and W. Yuan, Dual spaces of anisotropic mixed-norm Hardy spaces, Proc. Amer. Math. Soc. 147 (2019), no. 3, 1201-1215.

[14] _ Atomic and Littlewood-Paley characterizations of anisotropic mixed-norm Hardy spaces and their applications, J. Geom. Anal. 29 (2019), no. 3, 1991-2067.

[15] _ Identification of anisotropic mixed-norm Hardy spaces and certain homogeneous Triebel-Lizorkin spaces, J. Approx. Theory 258 (2020), 105459, 27 pp.

[16] _ Real-variable characterizations of new anisotropic mixed-norm Hardy spaces, Commun. Pure Appl. Anal. 19 (2020), no. 6, 3033-3082.

[17] Y. Jiang, Average sampling and reconstruction of reproducing kernel signals in mixed Lebesgue spaces, J. Math. Anal. Appl. 480 (2019), no. 1, 123370, 14 pp.

[18] Y. Jiang and J. Kou, Semi-average sampling for shift-invariant signals in a mixed Lebesgue space, Numer. Funct. Anal. Optim. 41 (2020), no. 9, 1045-1064.

[19] Y. Jiang and J. Li, (p,q)-frames in shift-invariant subspaces of mixed Lebesgue spaces $L^{p, q}\left(\mathbf{R} \times \mathbf{R}^{d}\right)$, arXiv:2001.08519.

[20] Y. Jiang and W. Sun, Adaptive sampling of time-space signals in a reproducing kernel subspace of mixed Lebesgue space, Banach J. Math. Anal. 14 (2020), no. 3, 821-841. 
[21] J. Johnsen, S. Munch Hansen and W. Sickel, Characterisation by local means of anisotropic Lizorkin-Triebel spaces with mixed norms, Z. Anal. Anwend. 32 (2013), no. $3,257-277$.

[22] _ Anisotropic Lizorkin-Triebel spaces with mixed norms-traces on smooth boundaries, Math. Nachr. 288 (2015), no. 11-12, 1327-1359.

[23] A. Kumar, D. Patel and S. Sampath, Sampling and reconstruction in reproducing kernel subspaces of mixed Lebesgue spaces, J. Pseudo-Differ. Oper. Appl. 11 (2020), no. $2,843-868$.

[24] R. Li, B. Liu, R. Liu and Q. Zhang, Nonuniform sampling in principal shift-invariant subspaces of mixed Lebesgue spaces $L^{p, q}\left(\mathbb{R}^{d+1}\right)$, J. Math. Anal. Appl. 453 (2017), no. 2, 928-941.

[25] - The $L^{p, q}$-stability of the shifts of finitely many functions in mixed Lebesgue spaces $L^{p, q}\left(\mathbb{R}^{d+1}\right)$, Acta Math. Sin. (Engl. Ser.) 34 (2018), no. 6, 1001-1014.

[26] A. Stefanov and R. H. Torres, Calderón-Zygmund operators on mixed Lebesgue spaces and applications to null forms, J. London Math. Soc. (2) 70 (2004), no. 2, 447-462.

[27] R. H. Torres and E. L. Ward, Leibniz's rule, sampling and wavelets on mixed Lebesgue spaces, J. Fourier Anal. Appl. 21 (2015), no. 5, 1053-1076.

[28] M. Unser, Sampling-50 years after Shannon, Proc. IEEE 88 (2000), no. 34, 569-587.

[29] J. Yang, Random sampling and reconstruction in multiply generated shift-invariant spaces, Anal. Appl. (Singap.) 17 (2019), no. 2, 323-347.

[30] Q. Zhang, Reconstruction of functions in principal shift-invariant subspaces of mixed Lebesgue spaces, arXiv:1801.05715.

[31]_ Nonuniform average sampling in multiply generated shift-invariant subspaces of mixed Lebesgue spaces, Int. J. Wavelets Multiresolut. Inf. Process. 18 (2020), no. 3, 2050013, 16 pp.

Yingchun Jiang and Jiao Li

School of Mathematics and Computational Science, Guilin University of Electronic Technology, Guilin, 541004, China

E-mail addresses: guilinjiang@126.com, lijiao171328@163.com 